\title{
Curcumin modulation of the activation of PYK2 in peripheral blood mononuclear cells from patients with lupus nephritis
}

\author{
Meiying Wang ${ }^{1,2}$, Gengmin Zhou ${ }^{1}$, Jiyang Lv $^{1}$, Peiying Zeng ${ }^{1}$, Chengshan Guo ${ }^{2}$, Qingwen Wang ${ }^{1}$ \\ ${ }^{1}$ Department of Rheumatology and Immunology, Peking University Shenzhen Hospital, Shenzhen, China \\ 2Department of Rheumatology and Immunology, The People's Hospital of Bao'an District, Shenzhen City, China
}

\begin{abstract}
Introduction: Proline-rich tyrosine kinase 2 (PYK2) provides important signals during the activation of lymphocytes, which is essential in autoimmune diseases. Systemic lupus erythematosus (SLE) is a representative autoimmune disease, and lupus nephritis (LN) is one of its most severe complications. Although glucocorticoid-binding immuno-suppression is the first-line therapy for patients with LN, the common and severe side effects of such treatment call for new strategies to improve long-term prognosis and life quality for these patients. Curcumin has been used to treat autoimmune disease with good curative effect, but little is known about the effect of curcumin on LN patients. Our aim was to investigate the mechanism of curcumin for management of LN, specifically regarding the PYK2 pathways.

Material and methods: Freshly isolated peripheral blood mononuclear cells (PBMCs) from 20 LN patients and 20 healthy individuals were cultured and stimulated with either PMA, PMA+TyrA9 (PYK2 specific inhibitor), or PMA+Curcumin, and with PBS as control. After 48 hours of incubation, cells were harvested and the expression of PYK2, p-PYK2, CD40L, CTLA-4, and PBMCs proliferation were measured. Then the expression and activation of PYK2 was evaluated using Western blot, the expression of costimulatory molecules CD40L and CTLA-4 protein was evaluated using flow cytometry, and PBMC proliferation was assessed using a $\left[{ }^{3} \mathrm{H}\right]$-thymidine incorporation assay.

Results: Curcumin inhibited the expression and activation of PYK2 in PBMCs in patients with LN in vitro. The inhibition rate of curcumin was negatively correlated with the level of serum complement, but positively correlated with 24-h proteinuria. Curcumin also suppressed the expression of costimulatory molecules CD4OL and CTLA-4, as well as PBMC proliferation. Interestingly, these effects were not reproduced on PBMC cultures of healthy subjects.

Conclusions: The inhibition of PYK2 signalling protein may be one of the mechanisms underlying the action of curcumin in LN treatment.
\end{abstract}

Key words: lupus nephritis, PYK2, CD4OL, CTLA-4, curcumin.

\section{Introduction}

Systemic lupus erythematous (SLE) is associated with multiple organ involvement. Among the several manifestations of SLE, renal involvement, known as lupus nephritis $(\mathrm{LN})$, is a common and severe complication and a major predictor of poor outcome [1]. Glucocorticoids and immunosuppressive drugs can inhibit immune response in numerous cases and are the standard of care for SLE patients, particularly for those with organ damage (such as LN) [2]. However, common and severe side effects associated with treatment with glucocorticoids and immunosuppressive

Address for correspondence:

Meiying Wang and Qingwen Wang Department of Rheumatology and Immunology, Peking University Shenzhen Hospital, No. 1120 Road, Futian District, Shenzhen 518036, China, e-mail: wmy99wmy99@163.com (Wang My) and wgw_sw@163.com (Wang Q)

Submitted: 22.09.2017; Accepted: 1.12.2017 
agents (for example, infection, osteoporosis, and peptic ulcers) call for safer and more tolerable therapeutic alternatives for LN patients.

Curcumin is a polyphenolic compound derived from the roots of the plant Curcuma longa, which is used in traditional Asian medicine $[3,4]$. Curcumin has been shown to exhibit immunomodulatory properties in lots of diseases [5, 6]. A previous study showed that curcumin can modulate the cellular response and growth of cells within the immune system [7]. In addition, a recent report showed that curcumin modulates Th17/Treg balance specifically on CD4+ T cells of SLE patients without affecting healthy subjects [8]. However, the precise molecular mechanism for such effects of curcumin in LN therapy is still unclear despite the extent of curcumin's effects on various biological processes.

The expression of costimulatory molecules such as CD4OL and CTLA-4, which are essential for lymphocyte activation [9], is up-regulated in human lupus T cells [10] or in patients with SLE [11]. Lupus mice treated with monoclonal antibody against CD4OL or CTLA4-Ig showed low levels of anti-double-stranded DNA antibodies, delayed development of nephritis, and prolonged survival time [12, 13]. Therefore, CD4OL and CTLA-4 become targets in considering effective therapeutic strategies for $\mathrm{LN}$.

Proline-rich tyrosine kinase 2 (PYK2) is a non-receptor protein tyrosine kinase belonging to the adhesion kinase superfamily [14]. Previous reports showed that PYK2 is over expressed in glomeruli from human and mice crescentic glomerulonephritis, and its over expression is closely associated with the onset of glomerular nephritis [15]. Moreover, the activation of the PYK2 signalling on rheumatoid arthritis (RA) lining cells plays a pivotal role in inflammatory cell adhesion and migration into the synovial tissue lesions [16]. Treatments targeting the PYK2 signalling pathway may be effective in autoimmune diseases.

Studies over the years have shown that the LPS-stimulated phosphorylation of PYK2 in human tracheal smooth muscle cells is blocked by pretreatment with curcumin [17]. In addition, the hyperosmotic-stress-induced rapid phosphorylation of PYK2 in rat aortic smooth muscle cells is blocked by curcumin [18]. However, the curcumin modulation of PYK2 phosphorylation in peripheral blood mononuclear cells (PBMCs) from LN patients remains unclear.

In the present study, the expression and activation of PYK2 in LN patients' PBMCs pretreated by curcumin in vitro was examined. Furthermore, the expression of costimulatory molecules CD4OL and CTLA-4, as well as PBMC proliferation, inhibited by curcumin, was investigated.

\section{Materials and methods \\ Subjects of the study}

The study subjects were 20 healthy volunteers (5 men and 15 women, mean age 38.4 years [range 23-64 years]), and $20 \mathrm{LN}$ patients (three men and 17 women, mean age 34.5 years [range $15-67$ years]). The diagnosis of SLE was confirmed according to the American College of Rheumatology criteria for systemic lupus erythematous, and the diagnosis of LN was confirmed in the presence of $>500 \mathrm{mg}$ per $24 \mathrm{~h}$ proteinuria on at least two occasions, as well as kidney biopsy [19]. The activity of SLE (SLEDAI) was assessed by a validated questionnaire for SLE disease activity [20]. All patients with confirmed LN were included.

Exclusion criteria were as follows: SLE patients with diabetes, urinary tract infection or urinary nephrolithiasis, patients with overlap connective tissue disease, vasculitis syndrome, SLE patients with antiphospholipid syndrome, end-stage renal disease, or patients on haemodialysis. Healthy volunteers were considered as the controls. The study protocol was approved by the Ethics Committee of Peking University Shenzhen Hospital, and informed consent was obtained from all patients and healthy subjects.

\section{Isolation and culture of PBMCs}

PBMCs from LN patients and healthy controls were purified from heparinised peripheral venous blood using Lymphoprep density gradient centrifugation (Invitrogen). The isolated PBMCs were suspended in buffered RPMI-1640 medium and then were divided into four groups: the first group $\left(1 \times 10^{6} / \mathrm{ml}\right)$ was cultured with $5 \mu$ phosphate-buffered saline (PBS) for $48 \mathrm{~h}$, as control, the second group $\left(1 \times 10^{6} / \mathrm{ml}\right)$ was cultured with $5 \mu \mathrm{l}(0.1$ $\mu \mathrm{g} / \mathrm{ml})$ PMA (Sigma) for $48 \mathrm{~h}$, and the third $\left(1 \times 10^{6} /\right.$ $\mathrm{ml})$ and the fourth group $\left(1 \times 10^{6} / \mathrm{ml}\right)$ were, respectively, pretreated with $1 \mu \mathrm{l}(0.1 \mu \mathrm{g} / \mathrm{ml})$ TyrA9 (Calbiochem) or $1 \mu \mathrm{l}(0.1 \mu \mathrm{g} / \mathrm{ml})$ curcumin (Shanghai Jinma Biotechnology, Shanghai, China) for one hour and then was continually cultured with $5 \mu \mathrm{l}$ PMA for $48 \mathrm{~h}$. These four groups were incubated in 24 -well culture dishes in $5 \% \mathrm{CO}_{2}$ at $37^{\circ} \mathrm{C}$.

\section{Measurement of PYK2 expression and activation using Western blot}

Cells were harvested and lysed for 30 min using cold RIPA buffer mixed with protease inhibitor compounds. The lysates were separated by a 3-16\% gradient gel, and then were transferred to PVDF membranes. Blots were blocked with $5 \%$ milk in TBST and probed for total PYK2, phospho-PYK2, or $\beta$-actin. Proteins were detected by a chemiluminescence detection system using Super 
Signal West Pico Substrate (Pierce). Antibodies used in cluded PYK2 (SC-9019) and p-PYK2 (SC-11767-R) (Sigma).

\section{Measurement of CD40L and CTLA-4 percentages using flow cytometry}

Cells were harvested and labelled with 5 ul FITC-conjugated anti-CD40L monoclonal antibody (TRAP1, BD Biosciences), FITC-conjugated anti-CTLA-4 monoclonal antibody (BNI3, BD BioSciences), or PE-conjugated

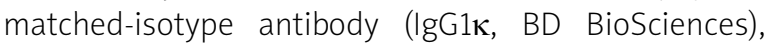
served as a control for $30 \mathrm{~min}$ at $4^{\circ} \mathrm{C}$, washed twice in PBS and subsequently analysed using FACScan (Becton-Dickinson, Mountain View, CA, USA). A minimum of $1 \times 10^{4}$ cells were counted and analysed for each determination. Results are expressed as the percentage of positive cells.

\section{Proliferation assays}

Harvested PBMCs $\left(2 \times 10^{5}\right.$ cells/well) were plated in 96-hole, flat-bottomed plates and then were pulsed for $18 \mathrm{~h}$ with $\left[{ }^{3} \mathrm{H}\right]$-thymidine $\left(1.0 \mu \mathrm{Ci} /\right.$ well). $\left[{ }^{3} \mathrm{H}\right]$-thymidine incorporation was measured using an LS6500 Microbeta Plus liquid scintillation counter (BACKMAN, U.S.). Each experiment was performed at least three times.

\section{Statistical analysis}

Values are shown as mean \pm standard deviation. Differences between two groups were conducted by $t$-test. The comparison among multiple groups was compared by ANOVA with Bonferroni test analysis. Correlations between two variables were analysed Spearman's rank test. The experimental data analyses were performed using SPSS16.0 statistical package; values of $p<0.05$ were considered statistically significant.

\section{Results}

\section{Curcumin reduces the expression and activation of PYK2 in PBMCs from patients with $\mathrm{LN}$ in vitro}

The levels of the expression and activation of PYK2 in PBMCs were analysed using Western blot as described in "Material and methods". As shown in Figure 1A (top blot), a marked reduction in PYK2 expression was observed in PBMCs following curcumin or TyrA9 pretreated in the LN patients but not in healthy donors. Quantitative analysis showed that the expression of PMA-stimulated PYK2 in PBMCs from LN patients was significantly up-regulated but was down-regulated by pretreatment with curcumin $(1.43 \pm 0.44$ vs. $0.81 \pm 0.29, p=0.002, t=$ $3.942)$ or TyrA9 $(1.43 \pm 0.44$ vs. $0.93 \pm 0.28, p=0.020, t=$
2.343). Curcumin or TyrA9 pretreatment showed no inhibitory effect in the cells of the control group (Fig. 1B).

PYK2 activation is integral to its downstream signalling. The activation status of PYK2 was assessed by Western blot with a PYK2 phospho-specific antibody. Consistently, PYK2 was weakly phosphorylated on the tyrosine 402 residue in PBMCs cultured with the medium, whereas a markedly higher signal for phosphorylated PYK2 ( $p$-PYK2) was observed in lanes of PBMCs treated with PMA, suggesting higher level of PYK2 activation. However, when cells were pretreated with curcumin or TyrA9 before PMA stimulus, a much thinner band corresponding to p-PYK2 was seen in lanes of the LN patients, but not in lanes of the control group (Fig. 1A, middle blot). This has also been further verified by quantitative analysis, as shown in Figure 1B. The expression level of P-PYK2 stimulated by PMA was blocked by pretreatment with curcumin $(1.37 \pm 0.44$ vs. $0.51 \pm 0.19, p=0.001, t=$ 4.027) or TyrA9 (1.37 \pm 0.44 vs. $0.59 \pm 0.20, p=0.025$, $t=1.926)$ in LN patients. Instead, curcumin or TyrA9 pretreatment showed no inhibitory effect in the cells of the control group (Fig. 1B). The comparison of the inhibitory rates between curcumin and TyrA9 on PMA-stimulated phosphorylation of PYK2 in LN PBMCs was also analysed, and no significant difference was found $(0.51 \pm 0.19$ vs. $0.59 \pm 0.20, p=0.17, t=0.263$ ).

\section{Correlation between the effect of curcumin on the activation of PYK2 and clinical indices in LN patients}

In the LN group, the inhibition rate of PYK2 activation caused by curcumin showed a negative correlation with the level of serum complements ( $p<0.01, r=-0.532$ ) and a positive correlation with quantity of 24-h urine protein ( $p<0.01, r=0.6707$ ) (Fig. $2 \mathrm{~A}$ and $2 \mathrm{~B}$ ). However, the rate of inhibition of PYK2 activation caused by curcumin in LN patients showed no correlation with the SLEDAI score (data not show).

\section{Curcumin inhibits the expression of CD40L and CTLA- 4 in LN patients}

As shown in Figure 3, PMA induced the up-regulation of CD4OL and CTLA-4 protein in PBMCs from LN patients and normal individuals. The expression levels of PMA-stimulated CD4OL and CTLA-4 protein in PBMCS from $L N$ patients were significantly reduced by pretreatment with curcumin [CD4OL (29.7 \pm 9.9 vs. $12.1 \pm 4.2, p=$ $0.008, t=3.845)$, CTLA-4 (25.7 \pm 6.9 vs. $12.9 \pm 4.2, p=0.011$, $t=3.057)$ ] or TyrA9 [CD40L (29.7 \pm 9.9 vs. $19.1 \pm 4.5, p=$ $0.014, t=2.861)$, CTLA-4 (25.7 \pm 6.9 vs. $14.1 \pm 3.9, p=0.019$, $t=2.447)$ ], but curcumin or TyrA9 pretreatment showed no inhibitory effect in the cells of the control group. 
A

Medium PMA

PMA + TyrA9 PMA + Cur
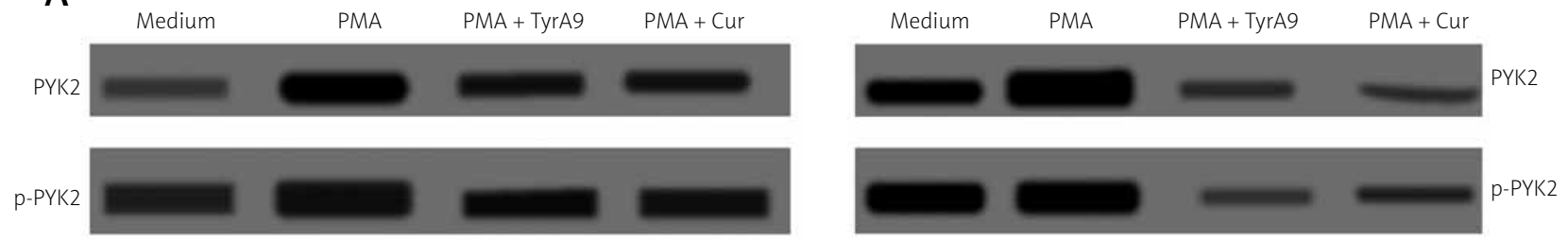

$\beta$-actin
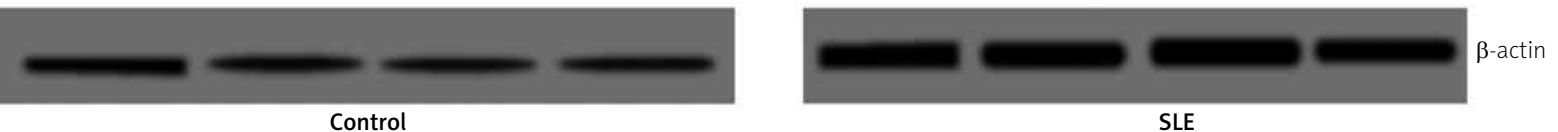

Control

SLE

B

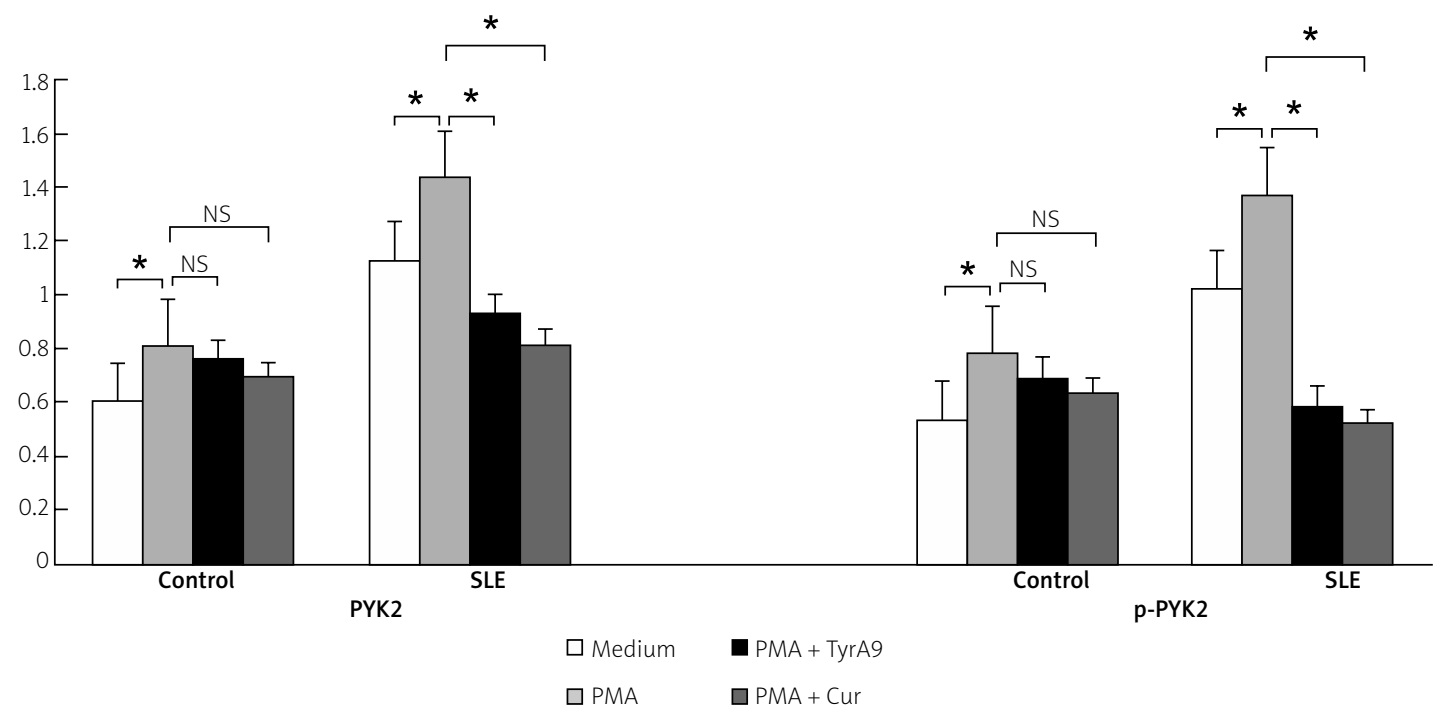

Fig. 1. Reduced expression and activation of PYK2 by curcumin in PBMCs from LN patients. A. Representative blot of total PYK2 protein and phosphorylated PYK2(p-PYK2) in PBMCs protein lysates obtained from normal individuals $(n=20)$ and LN patients $(n=20)$. Total proteins extracted from PBMCs lysates were run on gradient gel, and subsequently analysed for PYK2 and p-PYK2 as indicated in patients and methods. PMA induced a stronger band corresponding to PYK2 and P-PYK2 in PBMCs, curcumin pretreatment inhibited the expression of PMA-induced PYK2 and P-PYK2 in LN patients but not in normal individuals. B. Data for protein level were obtained by computerized analysis of the Western blots. The bars represent the mean and standard deviation of three separate experiments. Statistical comparison of each group was made to the corresponding PMA group. Significance $\left(^{*}\right)$ was set to $p<0.05$.

\section{Curcumin pretreatment inhibits the proliferation of PBMCs in LN patients}

As expected, the proliferation of PBMCs from normal individuals in vitro was weaker than that of LN patients. With stimulation by PMA, the proliferation of PBMCs from LN patients and normal individuals was significantly up-regulated. The PMA-stimulated proliferation of PBMCs from LN patients was significantly inhibited by pretreatment with curcumin $(15000 \pm 6263$ vs. 5498 $\pm 1707, p<0.001, t=4.732)$ or TyrA9 (15 $000 \pm 6263$ vs. $6000 \pm 2181, p<0.001, t=4.185)$. However, curcumin or TyrA9 pretreatment showed no inhibitory effect in the proliferation of PBMCs from the control group (Fig. 4).

\section{Discussion}

In this study, we showed that curcumin inhibited the expression and activation of PYK2 in PBMCs from LN patients. The rate of inhibition was negatively correlated with the level of serum complement and significantly positively correlated with $24-h$ proteinuria. Isolation and culturing of PBMCs showed that curcumin suppressed the expression of costimulatory molecules CD4OL and CTLA-4, as well as PBMCs proliferation. Our results demonstrated for the first time that curcumin can modulate the activation of PYK2 signalling, which provides us with new insight into the immunoregulatory mechanisms of curcumin for the treatment of LN. 
A

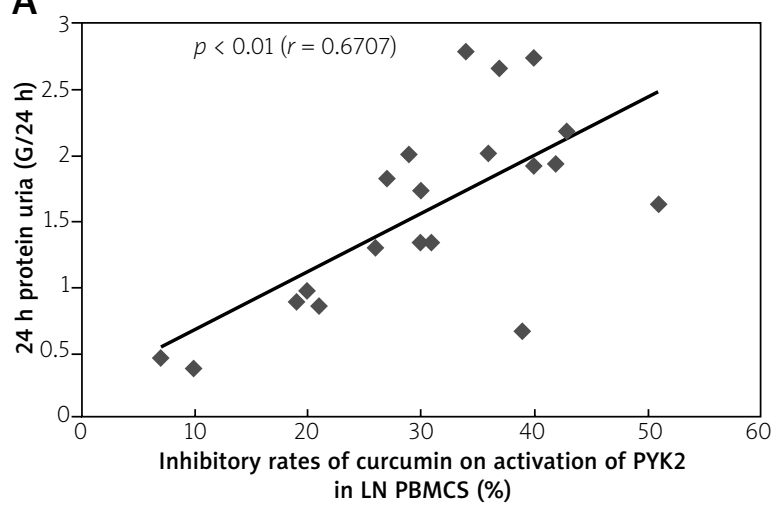

B

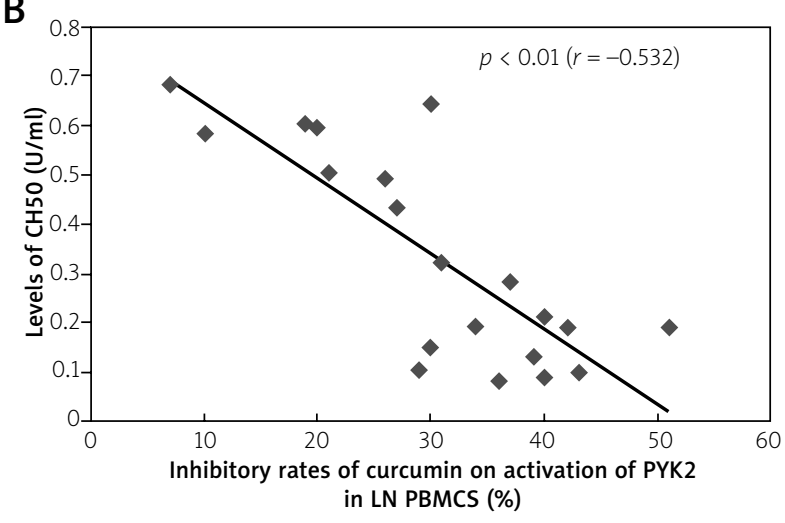

Fig. 2. Correlation between inhibition rates of curcumin on the activation of PYK2 and 24 hour proteinuria or levels of $\mathrm{CH} 50$ in LN patients. A. The inhibition rate of PYK2 activation caused by curcumin showed a positive correlation with quantity of $24 \mathrm{~h}$ urine protein. $\mathrm{B}$. The inhibition rate of PYK 2 activation caused by curcumin showed a negative correlation with the level of serum complements.

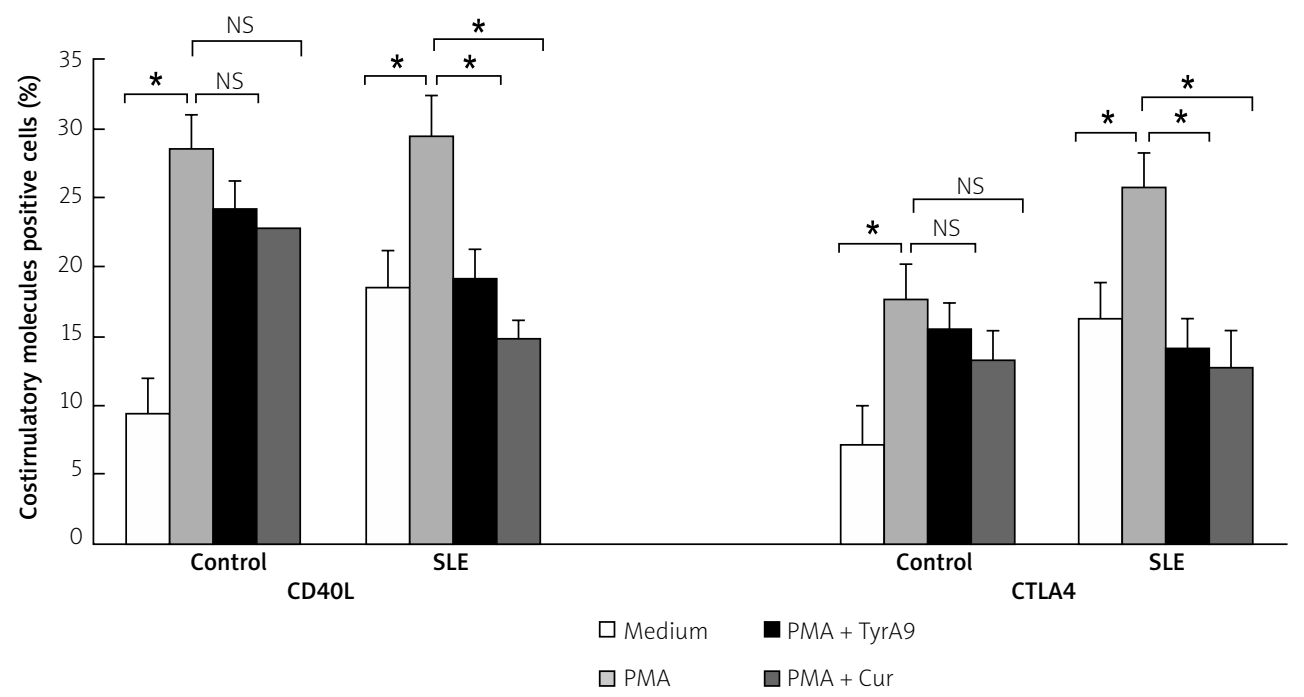

Fig. 3. Down-regulation of CD4OL and CTLA-4 by curcumin in PBMCs from LN patients. PBMCs were incubated and analyzed for the expression of CD4OL and CTLA-4 by flow cytometric analysis as indicated in patients and methods.Curcumin pretreatment inhibited the expression of PMA-induced CD4OL and CTLA-4 in LN patients but not in normal individuals. All experiments were repeated at least three times with similar results. Statistical comparison of each group was made to the PMA group. Significance $\left(^{*}\right)$ was set to $p<0.05$.

Although curcumin has been extensively used in traditional medicine in clinical practice for the treatment of rheumatoid arthritis [21], ulcerative colitis [22], arteriosclerosis [23], skin lesions [24], hepatic [25] and biliary disorders [26], and others, its anti-inflammatory action has not been fully understood. Recent studies have suggested that the inhibition of proliferation of $\mathrm{T}$ lymphocytes may be responsible for the anti-inflammatory action of curcumin $[27,28]$.

Another recent report showed that curcumin can modulate Th17/Treg balance specifically on CD4+ T cells of SLE patients without affecting healthy subjects [8]. Our results showed that curcumin could significantly suppress the expression and activation of PYK2 in PBMCs induced by PMA in LN patients. Our results also showed a significant negative correlation between the inhibition rate of curcumin on PYK2 and serum $\mathrm{CH} 50$ in LN patients. On the contrary, a positive correlation between the inhibition rate of curcumin on PYK2 and 24-h proteinuria was found for the total cohort of LN patients. The results were consistent with the report that LPS-induced PYK2 phosphorylation was blocked by pretreat- 


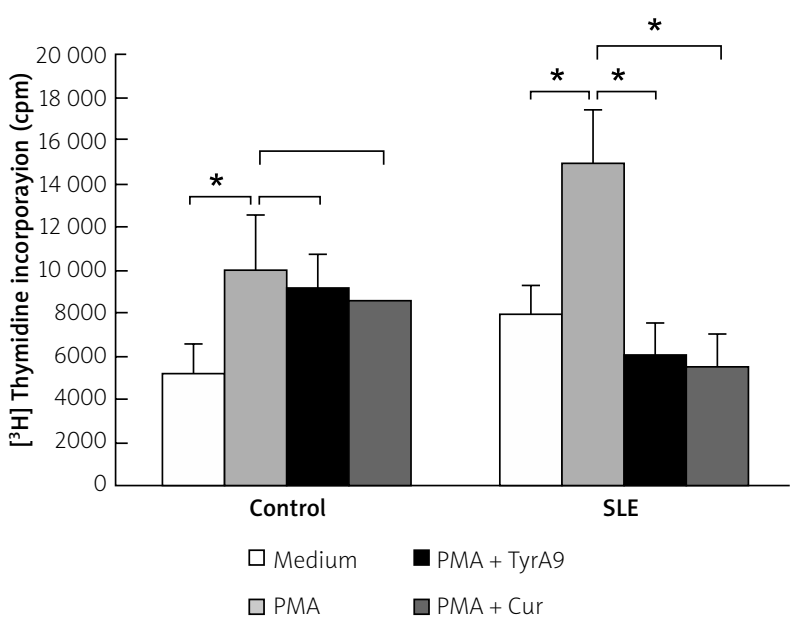

Fig. 4. Curcumin inhibits the proliferation of PBMCs in LN patients. Isolated PBMCs were cultured as indicated in patients and methods, and then cultures were pulsed with $\left[{ }^{3} \mathrm{H}\right]$-thymidine $(1.0 \mu \mathrm{Ci} /$ well $)$ for $18 \mathrm{~h}$ before harvesting the cells. Subsequently, $\left[{ }^{3} \mathrm{H}\right]$-thymidine incorporation was measured. PMA induced the proliferation of PBMCs from each group. Instead, curcumin pretreatment inhibited the PMA-stimulated proliferation of PBMCs in LN patients but not in normal individuals. Statistical comparison of all groups was made to the PMA group. Significance $\left(^{*}\right)$ was set to $p<0.05$.

ment with curcumin [17]. These findings suggest that the inhibition of activation of PYK 2 may be implicated in the mechanism(s) of curcumin in the treatment of SLE, especially for $L N$.

Previous studies revealed that curcumin can effectively treat experimental colitis by inhibiting the expression levels of CD4OL in CD8+ CD1c+ cells [29]. CD40L is essential for lymphocyte activation in LN patients; therefore, this costimulatory molecule eventually becomes the target in considering effective strategies in the treatment of these patients [30]. Our results showed that curcumin could inhibit the expression of CD4OL in PBMCs from LN patients. Consistent with the decreased CD40L expression, the proliferation of PBMCs in LN patients was also reduced by curcumin.

With regard to the declining expression of CTLA-4, we speculated that the inhibitory effects of curcumin on T-cell activation may be involved in the process. As we know, CTLA-4 is mainly expressed in active $T$ cells, and its peak appears at 24 hours after activation of T cells, but it was virtually undetectable on resting $T$ cells [31]. In our study, curcumin inhibited the proliferation of lymphocytes, and then the resting $T$ cells increased accordingly, which might be one of the causes for the decrease of CTLA-4 expression. In addition, different doses of curcumin can cause different reactions in lymphocytes [8, 32]. A published paper [32] reported that high-dose curcumin $(20 \mu \mathrm{M})$ significantly up-regulated CTLA-4 expression in a dose-dependent manner. However, a recent report showed [8] that low-dose curcumin $(0.1-1 \mu \mathrm{g} / \mathrm{ml})$ can promote the apoptosis of $\mathrm{T}$ lymphocytes, but high-dose of curcumin $(100 \mu \mathrm{g} / \mathrm{ml})$ can inhibit the apoptosis of $\mathrm{T}$ lymphocytes. Our results also found that low-doses of curcumin $(0.1 \mathrm{\mu g} / \mathrm{ml})$ were needed to inhibit the activation of lymphocytes and to decrease the expression of CTLA-4.

Interestingly, we found that the ability of low-dose curcumin to modulate PYK2 activation is only apparent in PBMCs cultures of SLE patients without affecting healthy subjects. Another study also found that lowdose curcumin was not able to modulate Th17/Treg balance on CD4+ T cells of healthy subjects [8]. Differences in dose response shown in CD4+ T cells of SLE patients and healthy subjects indicated that curcumin may be more sensitive to enter CD4+ T cells from SLE patients than from healthy subjects. Limitations of the present study include the lack of experiments for the assessment of the precise pathway regulated by different doses curcumin in order to modulate the activation of PYK2 in LN patients. In the follow-up experiment, we will continue to improve the exact mechanism of curcumin inducing lymphocyte apoptosis.

\section{Conclusions}

The results of this present study indicate that curcumin at low doses $(0.1 \mathrm{\mu g} / \mathrm{ml})$ can reduce the proliferation of PBMCs in LN patients by inhibiting the activation of PYK2. This ability to modulate PYK2 activation is specifically present only on PBMCs of SLE patients without affecting PBMCs of healthy subjects.

\section{Acknowledgments}

We thank LetPub for their assistance in editing the manuscript.

This work was supported by Grants from the Science and Technology Innovation Fund of Shenzhen, China (No. JCYJ20160427190358849, No. JCYJ20170307112009204), and the Medical Science and Technology Fund of Guangdong Province, China (No. A2016296).

The authors declare no conflict of interest. 


\section{References}

1. Paley MA, Strand V, Kim AH. From mechanism to therapies in systemic lupus erythematosus. Curr Opin Rheumatol 2017; 29: 178-186.

2. Jordan N, D'Cruz D. Current and emerging treatment options in the management of lupus. Immunotargets Ther 2016; 5: 9-20.

3. Abarikwu SO, Akiri OF, Durojaiye MA, Alabi AF. Combined administration of curcumin and gallic acid inhibits gallic acid-induced suppression of steroidogenesis, sperm output, antioxidant defenses and inflammatory responsive genes. J Steroid Biochem Mol Biol 2014; 143: 49-60.

4. Jeong CW, Yoo KY, Lee SH, et al. Curcumin protects against regional myocardial ischemia/reperfusion injury through activation of RISK/GSK-3 and inhibition of p38 MAPK and JNK. J Cardiovasc Pharmacol Ther 2012; 17: 387-394.

5. Nayak AP, Mills T, Norton I. Lipid Based Nanosystems for Curcumin: Past, Present and Future. Curr Pharm Des 2016; 22 : 4247-4256.

6. Jagetia GC, Aggarwal BB. “Spicing up"of the immune system by curcumin. J Clin Immunol 2007; 27: 19-35.

7. Shirley SA, Montpetit AJ, Lockey RF, Mohapatra SS. Curcumin prevents human dendritic cell response to immune stimulants. Biochem Biophys Res Commu 2008; 374: 431-436.

8. Handono K, Pratama MZ, Endharti AT, Kalim H. Treatment of low doses curcumin could modulate Th17/Treg balance specifically on CD4+ T cell cultures of systemic lupus erythematosus patients. Cent Eur J Immunol 2015; 40: 461-469.

9. Pollard KM, Arnush M, Hultman P, Kono DH. Costimulation requirements of induced murine systemic autoimmune disease. J Immunol 2004; 173: 5880-5887.

10. Yi Y, McNerney M, Datta SK. Regulatory defects in Cbl and mitogen-activated protein kinase (extracellular signal-related kinase) pathways cause persistent hyperexpression of CD40 ligand in human lupus T cells. J Immunol 2000; 165: 6627-6634.

11. Wong CK, Lit LC, Tam LS, et al. Aberrant production of soluble costimulatory molecules CTLA-4,CD28,CD80 and CD86 in patients with systemic lupus erythematosus. Rheumatology (Oxford) 2005; 44: 989-994.

12. Mohan C, Shi Y, Laman JD, Datta SK. Interaction between CD40 and its ligand gp39 in the development of murine lupus nephritis. J Immunol 1995; 154: 1470-1480.

13. Finck BK, Linsley PS, Wofsy D. Treatment of murine lupus with CTLA4Ig. Science 1994; 265: 1225-1227.

14. Sasaki H, Nagura K, Ishino M, et al. Cloning and characterization of cell adhesion kinase, a novel protein-tyrosine kinase of the focal adhesion kinase subfamily. Journal of Biological Chemistry 1995; 270: 21206-21219.

15. Takagi C, Ueki K, Ikeuchi H, et al. Increased expression of cell adhesion kinase beta in human and rat crescentic glomerulonephritis. Am J Kidney Dis 2002; 39: 174-182.

16. Shahrara S, Castro-Rueda HP, Haines GK, Koch AE. Differential expression of the FAK family kinases in rheumatoid arthritis and osteoarthritis synovial tissues. Arthritis Res Ther 2007; 9: R112.
17. Lin WN, Luo SF, Wu CB, et al. Lipopolysaccharide induces VCAM-1 expression and neutrophil adhesion to human tracheal smooth muscle cells: involvement of Src/EGFR/ PI3-K/ Akt pathway. Toxicol Appl Pharmacol 2008; 228: 256-268.

18. Koh YH, Che W, Higashiyama $\mathrm{S}$, et al. Osmotic stress induces HB-EGF gene expression via $\mathrm{Ca}(2+) / \mathrm{Pyk} 2 / \mathrm{JNK}$ signal cascades in rat aortic smooth muscle cells. J Biochem 2001; 130: 351358.

19. Tan EM, Cohen AS, Fries JF, et al. The 1982 revised criteria for the classification of systemic lupus erythematosus. Arthritis Rheum 1982; 25: 1271-1277.

20. Bombardier C, Gladman DD, Urowitz MB, et al. Derivation of the SLEDAI: a disease activity index for lupus patients. Arthritis Rheum 1992; 35: 630-640.

21. Arora R, Kuhad A, Kaur IP, Chopra K. Curcumin loaded solid lipid nanoparticles ameliorate adjuvant-induced arthritis in rats. Eur J Pain 2015; 19: 940-952.

22. Gupta SC, Patchva S, Aggarwal BB. Therapeutic roles of curcumin: lessons learned from clinical trials. AAPS 2013; 15: 195-218.

23. Chen FY, Zhou J, Guo N, et al. Curcumin retunes cholesterol transport homeostasis and inflammation response in $\mathrm{M} 1$ macrophage to prevent atherosclerosis. Biochem Biophys Res Commun 2015; 467: 872-878.

24. Castangia I, Nácher A, Caddeo C, et al. Fabrication of quercetin and curcumin bionanovesicles for the prevention and rapid regeneration of full-thickness skin defects on mice. Acta Biomate 2014; 10: 1292-1300.

25. Lee GH, Lee HY, Choi MK, et al. Protective effect of Curcuma longa L. extract on CCl4- induced acute hepatic stress. BMC Res Notes 2017; 10: 77.

26. Tokac M, Taner G, Aydin S, et al. Protective effects of curcumin against oxidative stress parameters and DNA damage in the livers and kidneys of rats with biliary obstruction. Food Chem Toxicol 2013; 61: 28-35.

27. Kim G, Jang MS, Son YM, et al. Curcumin inhibits CD4(+) T cell activation, but augments CD69 expression and TGF- $\beta 1$-mediated generation of regulatory T cells at late phase. PLoS On 2013; 8: e62300.

28. Sebastià N, Soriano JM, Barquinero JF, et al. In vitro cytogenetic and genotoxic effects of curcumin on human peripheral blood lymphocytes. Food Chem Toxicol 2012; 50: 3229-3233.

29. Zhao HM, Han F, Xu R, et al. Therapeutic effect of curcumin on experimental colitis mediated by inhibiting CD8+CD11c+ cells. World J Gastroenterol 2017; 23: 1804-1815.

30. Merrill JT. Co-stimulatory molecules as targets for treatment of lupus. Clin Immunol 2013; 148: 369-375.

31. Linsley PS, Greene JL, Tan P, et al. Coexpression and functional cooperation of CTLA-4 and CD28 on activated T lymphocytes. J Exp Med 1992; 176: 1595-1604.

32. Sharma S, Chopra K, Kulkarni SK, Agrewala JN. Resveratrol and curcumin suppress immune response through CD28/CTLA-4 and CD80 co-stimulatory pathway. Clin Exp Immunol 2007; 147: 155-163. 